\title{
Algunos componentes del estado de salud de la mujer cubana
}

\author{
Some components of the health status of the Cuban woman
}

\author{
Violeta R. Herrera Alcázar' ; Rosa María Torres Vidal" \\ 'Especialista de II Grado en Ginecología y Obstetricia. Máster en Atención Primaria \\ de Salud y Atención Integral a la Mujer. Escuela Nacional de Salud Pública. La \\ Habana, Cuba. \\ "Especialista de I Grado en Bioestadística. Máster en Estudios de Población. Escuela \\ Nacional de Salud Pública. La Habana, Cuba.
}

\section{RESUMEN}

Este trabajo está basado en datos estadísticos provenientes de sistemas de información estadísticos oficiales existentes. La proporción de mujeres en la población en el año 2007 era de 49,93 \% y existe un envejecimiento puesto que el grupo de 60 años y más que representaba el 9,40 \% en 1995 ascendió a 17,11\% en 2007. La natalidad y fecundidad mantuvieron una tendencia descendente y para el 2007 era de $10,0 \times 1000$ habitantes y 37,3 x 1000 mujeres de la edad entre 15-49 años, respectivamente. Hubo un descenso mantenido de las tasas de abortos inducidos en mujeres de 12-49 años desde 1990 que era de 45,6 a 20,4 x 1000 mujeres en el 2007, así como una elevada cobertura anticoncepcional de 77 \%. Las cinco primeras causas de mortalidad se mantienen: enfermedades del corazón, tumores malignos, enfermedades cerebrovasculares, influenza y neumonía y accidentes. La incidencia de tumores malignos tiene un comportamiento ascendente, su localización más frecuente es la mama. Más de $80 \%$ de los casos de cáncer cérvico uterino se diagnosticaron en etapa 0 , pero hubo un incremento de mujeres diagnosticadas en etapas más avanzadas. Las mujeres representaban el $19,5 \%$ de casos seropositivos (VIH/SIDA). La incidencia de blenorragia era mayor que la de sífilis con tasas respectivas de 31,8 y 12,7 por 100000 mujeres en el año 2007, siendo los jóvenes los más afectados. Los datos expuestos permiten una aproximación al estado de salud de la mujer cubana en los últimos 50 años. 
Palabras clave: Salud, mujer, mortalidad, morbilidad.

\begin{abstract}
This paper was based on statistical data from the existing official statistical information systems. The rate of women in the Cuban population in 2007 was $49.93 \%$; aging was present since the 60 years and over age group, which represented $9.40 \%$ in 1995 , rose to $17.11 \%$ in 2007 . Birth and fecundity rates kept their rising tendency amounting to $10 \times 1000$ pop. and $37.3 \times 1000$ women aged 15-49 years respectively in 2007 . There was a sustained drop in induced abortion rates in the 12-49 years group, from $45.6 \times 1000$ women in 1990 to $20.4 \times 1000$ women in 2007 as well as high contraception coverage of $77 \%$. The five first causes of mortality continued to be heart diseases, malignant tumors, cerebrovascular diseases, influenza and pneumonia, and accidents. The incidence of malignant tumors was on the rise and its more frequent location was the breast. Over $80 \%$ of uterine and cervix cancer cases was diagnosed in staging 0 , but the number of women diagnosed with this type of cancer in more advanced staging increased. The women accounted for $19.5 \%$ of seropositive cases (HIV/AIDS). The incidence rate of blenorrhagia was higher than that of syphilis (31.8 and 12.7 per 100000 women respectively) in 2007, being the youngest the most affected. The data provided here allows an approach to the health status of the Cuban women in the last 50 years.
\end{abstract}

Key words: Health, woman, mortality, morbidity.

\title{
I NTRODUCCI ÓN
}

A partir de 1959 ocurrieron en Cuba profundas transformaciones políticas, económicas y sociales que fueron cambiando el panorama general de la isla. En estas transformaciones estuvo muy presente la mujer, como actora y beneficiaria, comprendiendo el papel que le correspondía en la nueva sociedad donde mujeres y hombres serían beneficiados con la política de igualdad para todos seguida por el Gobierno Revolucionario, cuya voluntad ha sido la de proteger la salud de toda la población, y en particular la de la mujer y los niños. Lo anterior tiene su expresión en los diferentes programas sociales y en la legislación vigente y se aprecia en los indicadores de salud logrados en la actualidad.

No obstante los cambios sociales ocurridos en las últimas décadas que han posibilitado el incremento de la presencia de la mujer en esferas políticas, económicas y sociales del país, aún ellas siguen padeciendo las consecuencias de un patrón patriarcal establecido desde hace siglos, lo que se refleja en muchos aspectos de la vida cotidiana como es la distribución de las tareas dentro del hogar, la responsabilidad con los hijos pequeños, el acceso al poder, las posibilidades de superación y otras. Estas son manifestaciones de desigualdades entre los sexos que pudieran estar relacionadas con algunos problemas de salud en las distintas etapas del ciclo vital, independientemente de que hay condiciones y factores de riesgo o enfermedades exclusivas o más frecuentes en las mujeres que en los hombres. ${ }^{1-3}$ 
En el ámbito internacional, específicamente a partir de 1999, en el vigésimo primer período extraordinario de sesiones de la Asamblea General donde se adopta la Declaración para el Desarrollo de los Objetivos del Milenio, suscrita y aprobada por los gobiernos, se acuerda una serie de acciones que constituyen retos para los gobiernos y países en el camino de alcanzar un desarrollo humano sostenible. ${ }^{4,5}$ Así por ejemplo, y para citar solamente dos indicadores, para 2015 la proporción de partos asistidos por personal calificado debería alcanzar una media mundial de 90 $\%$ y situarse al menos en el 60 \% en los países con altas tasas de mortalidad materna, la cual debía, según las aspiraciones, disminuir en sus dos terceras partes. En Cuba se ha obtenido logros importantes en la esfera de la salud y especialmente en la salud de las mujeres, ahora explícitos en esta Declaración, los cuales se han mantenido a pesar de las dificultades socioeconómicas por las que ha atravesado el país a partir de la década de los años 90, y que no tienen diferencias sustanciales en las diferentes regiones. ${ }^{6-11}$

El presente trabajo está basado en datos estadísticos provenientes de los sistemas de información estadísticos oficiales existentes que permiten describir algunas características que han contribuido al estado de salud de la mujer cubana en el presente.

\section{POBLACIÓN}

La población femenina, en 1960 representaba el 48,52 \% del total de la población y en 1995, 2000, 2005 y 2007, el 49,72; 49,95; 49,92 y 49,93\%, respectivamente, lo que no muestra cambios proporcionales en cuanto a la composición por sexos de la población.

Sin embargo, y con el proceso de envejecimiento de la población ocurren cambios notables en el país en cuanto a la composición por edades. La población femenina de 60 años y más que en 1995 fue del 9,40\%, en el 2000 aumentó al 13,32 \% y ya en 2007 representaba el 17,11 \% del total de la población de féminas, lo que va a influenciar directamente sobre algunos padecimientos y enfermedades, ${ }^{3,12,13}$ además, y aunque el grupo en edad fértil no varía en el porcentaje con respecto a la población femenina, si existe progresivamente un desplazamiento ascendente hacia el grupo entre 35 y 44 años (35-39 y 40-44), lo que pudiera incidir en las enfermedades crónicas y condiciones de riesgo que se presentan durante la gravidez y sus posibles complicaciones (tabla 1).

\section{ESPERANZA DE VI DA AL NACER}

La esperanza de vida al nacer ha aumentado para ambos sexos, en las mujeres a 80,02 años en la etapa 2005-2007, lo cual también está en relación directa con la morbilidad y la mortalidad. Para ambos sexos se ve un enlentecimiento de este fenómeno en la década de los años 90 (tabla 2).

\section{NUPCI ALI DAD Y DI VORCI ALI DAD}


Después de un descenso en los años 90, la nupcialidad aumentó en los años 2006 y 2007. Sin embargo los divorcios diminuyeron en el año 2007 con respecto al 2006. Existe un aumento de los divorcios en la medida que aumentan los años de unión, lo que puede repercutir en el estado psicológico y emocional de estas mujeres que por lo demás ya no son las más jóvenes. ${ }^{14}$

\section{FECUNDI DAD}

En 1970 la natalidad era de 27,7 x 1000 habitantes y a partir de la década de los años 90 tuvo un descenso mantenido de 17,6 nacidos vivos por 1000 habitantes en 1990 a 10,0 en el 2007. Entre los factores que se mencionan para esto se encuentra el envejecimiento de las cohortes, el empoderamiento y en general, el desarrollo socioeconómico alcanzado por las mujeres (tabla 3 ).

Las provincias con tasas inferiores a 10 son: Ciudad de La Habana, Villa Clara, Matanzas, La Habana y Camagüey. Se mantiene la histórica menor incidencia de partos en los meses de abril, mayo y junio y su incremento en septiembre y octubre.

Los niveles de fecundidad general (TFG) disminuyen desde 1965 y de forma sostenida a partir de la década de los años 90 , de 48,0 por 1000 mujeres de 15 a 49 años en 1995, a 37,3 en el 2007. ${ }^{13,15}$

Las tasas específicas de fecundidad por edades muestran una máxima fecundidad entre 20 y 24 años y un ascenso en los grupos de 35-39 y 40-44, por razones de maternidad pospuesta que obedece a diversos aspectos del desarrollo sociocultural del país y que favorece y acerca a la fecundidad no temprana propia de países desarrollados (tabla 4).

La tasa global de fecundidad (TGF) -número de hijos promedio por mujer-, ha mantenido su declinación en los últimos años de 1,83 en 1990 a 1,58 en el 2000 y a 1,50 en el 2007. La tasa bruta de reproducción (TBR) -número de hijas promedio por mujer-, mantiene sus valores por debajo del nivel de reemplazo poblacional (al menos una hija por mujer) desde finales de la década de los 70 y en el año 2007 era de 0,71 . La provincia con más baja TBR en este año 2007 fue Matanzas, con 0,60 .

\section{ANTI CONCEPCIÓN}

La cobertura anticoncepcional en Cuba, al cierre del año 2007, era de 77,1 \% para el uso de métodos anticonceptivos modernos, los cuales son conocidos por la población, no obstante la la oferta en cuanto a su diversidad no es suficiente para la calidad de la anticoncepción (tabla 5 ).

La actual cobertura sitúa a Cuba entre los 23 países de más amplia cobertura anticoncepcional, todos desarrollados o de avanzado proceso de desarrollo. En Cuba se considera razonable los criterios de la OMS, que plantea no exceder el $35 \%$ de cobertura por DIU, dado que a partir de ahí se incrementa la intolerancia al método y se eleva el fallo por este que incrementa el embarazo no deseado y por ende el aborto. Se aspira al $35 \%$ para el 2010. También existen proyecciones para la diversificación de la anticoncepción desde la base del Sistema Nacional de Salud 
(SNS), es decir desde el consultorio médico, con una estrategia bien diseñada para promover la participación y responsabilidad del hombre ante la anticoncepción.

\section{ABORTOS I NDUCI DOS}

El aborto fue identificado como un problema de salud desde mediados de la década de los 60 por lo que se institucionalizó su práctica bajo ciertas regulaciones. Los servicios de aborto son seguros y dan cobertura a los abortos médicos y los voluntarios. Todas las formas de medir el comportamiento de los abortos muestran una disminución significativa en los últimos 15 años, lo que responde a una política dirigida a disminuir el evento basada en la educación sexual y la anticoncepción, que aún son insuficientes. Esta disminución se hace más cierta cuando la tasa se toma por 1000 mujeres de la edad entre 12 y 49 años $(20,4)$. También las regulaciones menstruales que se introducen extensivamente en el año 1993, mantienen un comportamiento con discreta tendencia a la disminución (tabla 6).

Estudios recientes del tema aborto demuestran que existe irresponsabilidad ante la prevención de un embarazo no deseado y confianza.... en que no le va a suceder... especialmente en jóvenes.

Existe en Cuba potencialidad para seguir diminuyendo el aborto voluntario, en cualquiera de sus formas, si se eleva la efectividad de la anticoncepción (hasta un $20 \%$ de abortos por fallos de anticoncepción) y si se adquiere la verdadera responsabilidad frente al embarazo no deseado. ${ }^{16,17}$

\section{ATENCIÓN AL EMBARAZO, PARTO Y PUERPERIO}

Durante la década de los años 90, el promedio de consultas fue superior a 10 por parto y en el año 2007 el promedio de consultas por parto se elevó a 12,6 y se mantiene en $99,9 \%$ el parto institucional. ${ }^{12,13}$

Existe una cobertura nacional de más 309 hogares maternos con una concepción diferente a la de sus inicios, donde se ingresaban las pacientes para acercarlas a las instituciones hospitalarias. En la actualidad, y a punto de partida de la situación económica de los años 90, en estas instituciones se mantiene a las gestantes que poseen un alto riesgo de tener un hijo con bajo peso. Esta y otras medidas han logrado que hoy el índice de bajo peso al nacer se conserve con su tendencia al descenso y en el 2007 fuera de 5,2 \%. Desde la década de los años 90 la tasa de mortalidad infantil se ha mantenido en niveles bajos comparados a países desarrollados, así en 1990 de 10,7; 1995, 9,4; 2000 7,2; 2005 6,2 y desde el 2006 es de $5,3 \times 1000$ nacidos vivos $(\mathrm{nv}){ }^{18}$

Si se tiene en cuenta que la cifra de mortalidad materna correspondiente al año 1959 era de 115,5 x 100000 nv, en el 2000 de 40,4 y en 2007 de 30,2 x 100000 nv, el descenso ha sido de 73,8 \%. Es notoria la reducción de la Razón de Muerte Materna (RMM) en Cuba en un período de 50 años a partir de 1959 y "es posiblemente la mayor lograda por un país latinoamericano en ese tiempo."19 Durante el año 2007 el 70,6 \% de las defunciones maternas correspondieron a causas directas y dentro de ellas los mayores niveles fueron por complicaciones relacionadas con el puerperio. 


\section{MORTALI DAD}

Las cinco primeras causas de muerte en la mujer fluctúan pero no varían desde hace varias décadas, y son: las enfermedades del corazón, los tumores malignos, la enfermedad cerebrovascular, la influenza y neumonía y los accidentes (tabla 7).

Es la enfermedad isquémica la que más aporta a la mortalidad por enfermedades del corazón $(127,1)$, le sigue la insuficiencia cardiaca $(20,5)$ y la enfermedad hipertensiva $(15,3)$, lo que está en concordancia con el mayor número de mujeres que arriban a edades avanzadas.

Los tumores malignos constituyen la segunda causa de muerte en Cuba desde hace más de tres décadas. Las tasas crudas de mortalidad presentan un comportamiento ascendente de 108,4 defunciones por 100000 mujeres en 1990 a 156,3 en el 2007 (44,2\% de incremento en el período).

En relación con todas las defunciones en el sexo femenino, los tumores malignos han ido aumentando proporcionalmente, en 1995 era de 17,7\% y ya en el 2006, de $22,9 \%$

Las principales localizaciones en orden decreciente durante el año 2007 correspondieron a: tráquea, bronquios y pulmón $(28,7)$, mama $(23,7)$ e intestino, excepto recto $(18,2)$. En un quinto lugar están los relacionados con el cuello del útero que aumenta su tasa de mortalidad de 5,7 en 1990 a 8,3 en el 2007 (tabla 8).

En el grupo de las enfermedades cerebrovasculares, las hemorrágicas son las más frecuentes, para una tasa de 34,1 x 1000000 mujeres. El incremento de la prevalencia de hipertensión arterial y la mortalidad por enfermedad hipertensiva resultan coherentes con este resultado.

Para estas tres primeras causas de muerte existen programas que desarrollan acciones preventivas que requieren de una mayor atención en los distintos niveles de salud, igual sucede con el cáncer del cuello uterino, cuyo programa de detección data de 1968.

Las enfermedades crónicas no trasmisibles y las muertes violentas (accidentes, lesiones auto inflingidas y agresiones) se ubican dentro de las cinco primeras causas de muerte para las mujeres en edad reproductiva, las últimas con más frecuencia entre las mujeres de 15-34 años.

\section{MORBI LI DAD}

\section{Tumores malignos}

Las principales localizaciones en orden decreciente correspondieron en el año 2007 a: cáncer de mama, piel, pulmón y cuello del útero (tabla 9).

Según datos obtenidos del Registro Nacional del Cáncer, la incidencia de tumores malignos en este grupo poblacional ha tenido un comportamiento ascendente 
durante la década de los noventa (40,9\% de incremento), para alcanzar la cifra de 232,0 por 100000 mujeres en 1999, en el que debe considerarse la mejoría del reporte de estas enfermedades. Este ha sido el comportamiento en la población general del país.

En Cuba se brinda atención especial al cáncer de cuello de útero desde 1968 a través del Programa Nacional para la Detección Precoz del Cáncer Cérvico Uterino y desde 1987 entró en marcha el Programa Nacional para la Detección Precoz del Cáncer de Mama. Más del $80 \%$ de los casos de cáncer cérvico uterino se diagnostican en etapa 0 , pero se observa un incremento de casos diagnosticados en etapas más avanzadas (tabla 10).

\section{I ncidencia de VI H/ SI DA}

En Cuba la prevalencia de VIH/SIDA se mantiene por debajo del 0,1 \% y la epidemia presenta un ritmo de crecimiento lento, pero sostenido La morbilidad en la mujer es más baja con respecto a los hombres, hasta el 2006 el 19,5\% de casos seropositivos era del sexo femenino. Hasta noviembre de 2007 se habían realizado más de 3,8 millones de pruebas de $\mathrm{VIH}$ a gestantes y la prevalencia reportada para el 2006 era de 0,01 \%. Dentro del Programa Materno Infantil tiene prioridad el problema VIH/SIDA y se mantiene una pesquisa activa para la prevención de la transmisión materno infantil. Entre otras medidas que han permitido elevar los niveles de diagnóstico precoz están la atención prenatal integral con acceso universal, la realización de la prueba de VIH ler. y 3er. trimestre, así como también a las parejas sexuales, la cesárea electiva y la lactancia artificial. Existe también un protocolo para la administración de antirretrovirales a la madre y al niño según los resultados de los estudios. Las mayores tasas las aportan los grupos de 20 a 24 años. Más de la mitad de los casos $(54,0 \%)$ corresponden a Ciudad de La Habana. ${ }^{20,21}$

\section{Sífilis y blenorragia}

Los grupos de edades más jóvenes y en plena edad reproductiva continúan siendo los más afectados por estas infecciones, lo cual se relaciona directamente con hábitos y conductas sexuales, correspondiendo la mayor incidencia a las jóvenes de 15 a 24 años.

La incidencia continúa siendo mayor para la blenorragia, con una tasa de 31,8 por 100000 mujeres durante el año 2007 , mientras que para la sífilis fue de $12,7 .{ }^{13}$

Se puede resumir que los datos expuestos permiten una aproximación al estado de salud de la mujer cubana en los últimos 50 años.

\section{REFERENCI AS BI BLI OGRÁFICAS}

1. Espín V. La mujer en Cuba: Historia. La Habana: Editorial de la Mujer; 1990.

2. Castañeda Abascal IE. Reflexiones teóricas sobre las diferencias en salud atribuibles al género. Rev Cubana Salud Pública [serie en Internet]. 2007 Jun [citado 14 Sep 2008 ];33(2). Disponible en: http://scielo.sld.cu/scielo.php?script=sci_arttext\&pid=S0864 $\underline{34662007000200011 \& \operatorname{lng}=\mathrm{es} \& \mathrm{n} r \mathrm{~m}=\text { iso }}$ 
3. Torres Vidal RM, Gran Álvarez MA. Panorama de salud de la mujer cubana. Rev Cubana Salud Pública [serie en Internet]. 2003 Sep [citado 28 Jul 2008];29 (3). Disponible en:

http://scielo.sld.cu/scielo.php?script=sci_arttext\&pid=S086434662003000300002

4. Human Development Report 2006. Statistics in the Human Development Report. [citado 23 Jul 2008]. Disponible en: http://hdr.undp.org/hdr2006/statistics/

5. Anuario estadístico de América latina y el Caribe. CEPAL [serie en Internet]. [citado 28 Jul. 2008]. Disponible en:

http://www.eclac.org/cgi-bin/getProd.asp? xml=/publicaciones/xml/3 28063/P28063.xml\&xsl=/deype/tpl/p9f.xsl

6. Rojas Ochoa F. Situación, sistema y recursos humanos en salud para el desarrollo en Cuba. Rev Cubana Salud Pública [serie en Internet]. 2003 Jun [citado 14 Sep 2008 ];29(2). Disponible en:

http://scielo.sld.cu/scielo.php?script=sci arttext\&pid=S0864 -

$\underline{34662003000200011 \& \operatorname{lng}=\mathrm{es} \& \mathrm{n} r \mathrm{~m}=\mathrm{iso}}$

7. López Pardo CM. El desarrollo humano y la equidad en Cuba: una visión actualizada. Rev Cubana Salud Pública [serie en Internet]. 2004 Mar [citado 14 Sep 2008]; 30(1). Disponible en:

http://scielo.sld.cu/scielo.php?script=sci arttext\&pid=S0864 -

$\underline{34662004000100006 \& \operatorname{lng}=\mathrm{es} \& \mathrm{n} r \mathrm{~m}=\mathrm{iso}}$

8. Ramírez Márquez A, López Pardo CM. A propósito de un sistema de monitoreo de la equidad en salud en Cuba. Rev Cubana Salud Pública [serie en Internet]. 2005 J un [citado 14 Sep2008]; 31(2). . Disponible en:

http://scielo. sld.cu/scielo.php?script=sci arttext\&pid=S0864-

$\underline{34662005000200002 \& \operatorname{lng}=\mathrm{es} \& \mathrm{n} r \mathrm{~m}=\mathrm{iso}}$

9. Situación de Salud de Las Américas. Indicadores Básicos 2004. Programa Especial de Análisis de Salud. Washington: Organización Panamericana de la Salud; 2006.

10. La salud en los objetivos de desarrollo del milenio. Organización Mundial de la Salud. [citado 28 Jul 2008]. Disponible en:

http://www.who.int/mdg/goals/041222health_mdgchart_sp.pdf

11. La OMS y los Objetivos de Desarrollo del Milenio Nota informativa $\mathrm{N}^{\circ} 290$. [citado 19 Sep 2008]. Disponible en:

http://www. who.int/mediacentre/factsheets/fs290/es/

12. Dirección Nacional de Estadísticas, Cuba. Anuario Estadístico de salud. La Habana: DNE; 2000.

13. Dirección Nacional de Estadísticas, Cuba. Anuario Estadístico de salud. La Habana: DNE; 2007.

14. Censo Nacional de Población y Viviendas. Cuba 2002. ONE. [citado 14 Sep 2008]. Disponible en:

http://www.cubagob.cu/otras_info/censo/graficos_mapas/anexo.htm

15. López Nistal L, Gran Álvarez MA, Felipe Ramos AM. Evolución de la fecundidad en Cuba en las últimas cinco décadas. Rev Temas Estadísticos de Salud [serie en 
Internet]. 2005 [citado $30 \mathrm{Jul}$ 2008]; 1(2). Disponible en: http://www.sld.cu/galerias/pdf/sitios/dne/nro2_fecundidad.pdf

16. Ministerio de Salud Pública de Cuba. Plan de Acción para la Reducción del Aborto Inseguro. La Habana: MINSAP; 2007.

17. Gran Álvarez MA. Interrupción voluntaria de embarazo y anticoncepción. Dos métodos de regulación de la fecundidad. Cuba. 1995-2000 [tesis]. La Habana: Escuela Nacional de Salud Pública; 2004.

18. Fondo de Población de las Naciones Unidas. Estado de la Población Mundial Mortalidad y morbilidad derivada de la maternidad. NewYork: FNUAP; 2000.

19. Cabezas Cruz E. Evolución de la mortalidad materna en Cuba. Rev Cubana Salud Pública [serie en Internet]. 2006 Mar [citado 14 Sep 2008 ]; 32(1). Disponible en: http://scielo.sld.cu/scielo.php?script=sci_arttext\&pid=S0864$\underline{34662006000100005 \& \operatorname{lng}=e s \& n r m=i s o}$

20. Colectivo de autores. Infecciones de Transmisión Sexual, pautas para su tratamiento. La Habana: MINSAP; 2004.

21. Resumen estadístico de la epidemia de VIH/SIDA en Cuba en 2007. Ministerio de Salud Pública. [citado 20 Sep 2008]. Disponible en:

http://www.sld.cu/servicios/sida/temas. php?idv=2240

Recibido: 6 de octubre de 2008.

Aprobado: 17 de octubre de 2008.

Violeta R. Herrera Alcázar. Escuela Nacional de Salud Pública. Calle Línea esq. I. El Vedado. La Habana 10400, Cuba.

E-mail: vioheral@infomed.sld.cu 
Tabla 1. Población femenina proyectada al 30 de junio según grupos de edad.

Años seleccionados

\begin{tabular}{|c|c|c|c|c|c|c|c|}
\hline Años & $<1$ aก̃o & $\begin{array}{c}\text { 1-4 } \\
\text { años }\end{array}$ & $\begin{array}{l}\text { 5-14 } \\
\text { años }\end{array}$ & $\begin{array}{l}\text { 15-49 } \\
\text { años }\end{array}$ & $\begin{array}{l}50-59 \\
\text { años }\end{array}$ & $\begin{array}{c}60 y+ \\
\text { años }\end{array}$ & Total \\
\hline 1995 & $\begin{array}{l}75358 \\
1,38 \%\end{array}$ & $\begin{array}{l}318802 \\
5,84 \%\end{array}$ & $\begin{array}{l}791131 \\
14,49 \%\end{array}$ & $\begin{array}{l}3021311 \\
55,35 \%\end{array}$ & $\begin{array}{l}738376 \\
13,53 \%\end{array}$ & $\begin{array}{l}513415 \\
9,40 \%\end{array}$ & 5458393 \\
\hline 2000 & $\begin{array}{l}67848 \\
1,21 \%\end{array}$ & $\begin{array}{l}295726 \\
5,29 \%\end{array}$ & $\begin{array}{l}843428 \\
15,09 \%\end{array}$ & $\begin{array}{l}3077713 \\
55,06 \%\end{array}$ & $\begin{array}{l}559986 \\
10,02 \%\end{array}$ & $\begin{array}{l}744479 \\
13,32 \%\end{array}$ & 5589180 \\
\hline 2007 & $\begin{array}{l}56962 \\
1,02 \%\end{array}$ & $\begin{array}{l}247227 \\
4,41 \%\end{array}$ & $\begin{array}{l}697742 \\
12,43 \%\end{array}$ & $\begin{array}{l}3012895 \\
53,68 \%\end{array}$ & $\begin{array}{l}637038 \\
11,35 \%\end{array}$ & $\begin{array}{l}960390 \\
17,11 \%\end{array}$ & 5612254 \\
\hline
\end{tabular}

Fuente: Proyección de la población. Nivel nacional y provincial. Período 1996-2010. Oficina Nacional de Estadisticas.

Tabla 2. Esperanza de vida al nacer. Períodos seleccionados

\begin{tabular}{|c|c|c|c|}
\hline Período & $\begin{array}{c}\text { Ambos } \\
\text { sexos }\end{array}$ & Varones & Hembras \\
\hline $1952-1954$ & 62,29 & 60,22 & 64,11 \\
\hline $1969-1971$ & 70,04 & 68,55 & 71,82 \\
\hline $1990-1991$ & 74,70 & 72,93 & 76,58 \\
\hline $1994-1995$ & 74,83 & 72,94 & 76,90 \\
\hline $1998-2000$ & 76,15 & 74,20 & 78,97 \\
\hline $2001-2003$ & 77,00 & 75,13 & 80,02 \\
\hline $2005-2007$ & 77,97 & & \\
\hline & & & \\
\hline & & & \\
\hline
\end{tabular}

Fuente: Oficina Nacional de Estadísticas. 
Tabla 3. Indicadores seleccionados sobre natalidad. Años seleccionados

\begin{tabular}{|r|r|r|r|r|}
\hline \multicolumn{1}{|l|}{} & \multicolumn{2}{|l|}{$\begin{array}{l}\text { Total de nacidos } \\
\text { Años }\end{array}$} & $\begin{array}{l}\text { Natalidad } \times 1000 \\
\text { vabitantes }\end{array}$ & \multicolumn{2}{l|}{$\begin{array}{l}\text { Nacidos vivos en } \\
\text { instituciones de salud }\end{array}$} \\
\hline 1970 & 237019 & 27,7 & 216926 & 96 \\
\hline 1990 & 186658 & 17,6 & 186254 & 99,8 \\
\hline 1995 & 147170 & 13,4 & 146944 & 99,8 \\
\hline 2000 & 143528 & 12,8 & 143355 & 99,9 \\
\hline 2005 & 120716 & 10,7 & 120587 & 99,9 \\
\hline $2007 *$ & 112472 & 10,0 & 112372 & 99,9 \\
\hline
\end{tabular}

*: Provisional.

Fuente: Anuario Estadístico de Salud. DNE. Año 2007.

Tabla 4. Tasa de fecundidad según edad de la madre (por 1000 mujeres de la edad). Años seleccionados

\begin{tabular}{|c|c|c|c|c|}
\hline Edad de la madre & 1995 & 2000 & 2005 & $2007^{*}$ \\
\hline $15-19$ & 60,2 & 49,6 & 44,9 & 44,6 \\
\hline $20-24$ & 91,4 & 96,5 & 93,0 & 90,8 \\
\hline $25-29$ & 78,8 & 89,2 & 81,3 & 75,4 \\
\hline $30-34$ & 46,8 & 55,0 & 53,2 & 49,5 \\
\hline $35-39$ & 17,1 & 22,4 & 21,9 & 21,8 \\
\hline $40-44$ & 2,5 & 3,8 & 4,1 & 3,9 \\
\hline $45-49$ & 0,5 & 0,2 & 0,2 & 0,2 \\
\hline Total & 48,0 & 47,4 & 40,1 & 37,3 \\
\hline
\end{tabular}

*: Provisional.

Fuente: ONE. Anuarios Demográficos de Cuba. 
Tabla 5. Estimación del porcentaje de cobertura anticoncepcional total y por métodos anticonceptivos.

Años seleccionados

\begin{tabular}{|c|c|c|c|c|c|c|c|c|}
\hline \multirow{2}{*}{ Años } & \multirow{2}{*}{$\begin{array}{c}\% \text { de } \\
\text { cobertura } \\
\text { total* }\end{array}$} & \multicolumn{7}{|c|}{ Porcentaje de cobertura anticoncepcional según métodos** } \\
\hline & & $\mathrm{DIU}^{* * *}$ & $\begin{array}{l}\text { Esterilización } \\
\text { femenina }\end{array}$ & Píldoras & Inyectables & Condón & Otros & Total \\
\hline 1995 & 72,0 & 65,0 & 20,0 & 7,5 & 1,0 & 5,0 & 1,5 & 100,0 \\
\hline 2000 & 73,0 & 60,0 & 20,0 & 6,8 & 2,0 & 9,0 & 2,2 & 100,0 \\
\hline 2005 & 77,1 & 60,2 & 19,8 & 7,2 & 1,8 & 9,6 & 1,4 & 100,0 \\
\hline 2007 & 77,1 & 56,0 & 19,6 & 9,0 & 2,6 & 11,4 & 1,4 & 100,0 \\
\hline
\end{tabular}

*: Porcentaje calculado sobre la base del total estimado de mujeres en edad fértil con actividad sexual que se declaran con pareja, **: porcentaje calculado para cada método, sobre la base del total estimado de mujeres en edad fértil con actividad sexual que se declaran con pareja y usan algún método anticonceptivo, $* * *$ : dispositivo intrauterino.

Fuente: DNE. Anuario Estadístico de Salud.

Tabla 6. Indicadores seleccionados sobre abortos inducidos, Años seleccionados

\begin{tabular}{|c|c|c|c|c|}
\hline \multicolumn{2}{|c|}{} & \multicolumn{2}{|c|}{ Abortos inducidos } \\
\hline \multicolumn{1}{|c|}{} & Total & Por 1 000 mujeres** & Por 100 partos & Por 100 embarazadas \\
\hline Años & 147530 & 45,6 & 78,3 & 43,9 \\
\hline 1990 & 83963 & 25,9 & 56,6 & 36,0 \\
\hline 1995 & 76293 & 23,0 & 52,7 & 34,5 \\
\hline 2000 & 66008 & 20,4 & 58,4 & 36,9 \\
\hline $2007^{*}$ & & & & \\
\hline
\end{tabular}

*: Provisional, **: mujeres entre 12 y 49 años de edad.

Fuente: DNE, Anuario Estadístico de Salud. 
Tabla 7. Principales causas de muerte en la mujer. Años seleccionados.

\begin{tabular}{|c|c|c|c|c|}
\hline \multirow[t]{2}{*}{ Causas } & \multicolumn{2}{|c|}{2000} & \multicolumn{2}{|c|}{$2007^{*}$} \\
\hline & Defunciones & Tasa & Defunciones & Tasa \\
\hline Enfermedades del corazón (I05 - I52) & 9214 & 164,9 & 10006 & 178,3 \\
\hline Tumores malignos (C00 - C97) & 70.51 & 126,2 & 8774 & 156,3 \\
\hline $\begin{array}{l}\text { Enfermedades cerebrovasculares } \\
\text { (I60-169) }\end{array}$ & 4220 & 75,5 & 4338 & 77,3 \\
\hline Influenza y neumonía (J09-J18) & 2295 & 47,3 & 2784 & 49,6 \\
\hline Accidentes (V01-X59, Y85-Y86) & 1938 & 34,7 & 1769 & 31,5 \\
\hline
\end{tabular}

*: Provisional.

Fuente: DNE. Anuario Estadístico de Salud.

Tabla 8. Mortalidad por cáncer cérvico uterino. Años seleccionados

\begin{tabular}{|c|c|c|c|}
\hline \multicolumn{1}{|c|}{ Años } & Defunciones & Tasa bruta** & Tasa ajustada*** \\
\hline 1990 & 301 & 5,7 & 5,0 \\
\hline 1995 & 339 & 6,2 & 5,0 \\
\hline 2000 & 395 & 7,1 & 5,6 \\
\hline 2005 & 459 & 8,2 & 5,6 \\
\hline $2007^{*}$ & 465 & 8,3 & 5,6 \\
\hline
\end{tabular}

*: Provisional, **: Tasa por 100000 mujeres, ***: Tasa ajustada por edad, población tipo la censal de 1981.

Fuente: DNE. Anuario Estadístico de Salud. 
Tabla 9. Incidencia de cáncer para sexo femenino y principales localizaciones.

2004

\begin{tabular}{|c|c|c|c|}
\hline Localización & No. & Tasa bruta* & Tasa ajustada** \\
\hline Mama & 2415 & 43,0 & 30,3 \\
\hline Piel & 2282 & 40,7 & 26,3 \\
\hline Pulmón & 1466 & 26,1 & 17,7 \\
\hline Cuello de útero & 1194 & 21,3 & 14,6 \\
\hline Colon & 1026 & 18,3 & 10,8 \\
\hline Cuerpo de útero & 436 & 7,8 & 5,5 \\
\hline Sistema hematopoyético & 398 & 7,1 & 5,5 \\
\hline Ovario & 396 & 7,1 & 5,3 \\
\hline Páncreas & 328 & 5,8 & 4,0 \\
\hline Recto & 327 & 5,8 & 3,6 \\
\hline Total & 13480 & 240,2 & 162,4 \\
\hline
\end{tabular}

*: Tasa por 100000 mujeres, **: Tasa ajustada por edad, población tipo censo 1981.

Fuente: DNE. Anuario Estadístico de Salud. 
Tabla 10. Casos positivos de cáncer cérvico uterino detectados en las mujeres examinadas por el programa según etapa clínica. Años seleccionados

\begin{tabular}{|c|c|c|c|c|c|c|}
\hline \multirow[t]{2}{*}{ Años } & \multicolumn{5}{|c|}{ Etapa clínica } & \multirow[t]{2}{*}{ Total * } \\
\hline & 0 & I & II & III & IV & \\
\hline 1990 & 989 & 107 & 23 & 4 & - & 1123 \\
\hline 1995 & 888 & 84 & 10 & - & - & 982 \\
\hline 2000 & 1171 & 171 & 42 & 2 & - & 1386 \\
\hline 2005 & 997 & 176 & 34 & 10 & - & 1217 \\
\hline $2007^{* *}$ & 1068 & 157 & 28 & 8 & 1 & 1262 \\
\hline \multicolumn{7}{|c|}{ Porcentaje del total } \\
\hline 1990 & 88,1 & 9,5 & 2,0 & 0,4 & - & 100,0 \\
\hline 1995 & 90,4 & 8,6 & 1,0 & - & - & 100,0 \\
\hline 2000 & 84,5 & 12,3 & 3,0 & 0,1 & - & 100,0 \\
\hline 2005 & 81,9 & 14,5 & 2,8 & 0,8 & - & 100,0 \\
\hline $2007^{* *}$ & 84,6 & 12,5 & 2,2 & 0,6 & 0,1 & 100,0 \\
\hline
\end{tabular}

*: Incluye etapa clínica sin clasificar, **: provisional.

Fuente: DNE del MINSAP. 